\title{
BMJ Open Screening for Hypertension in the INpatient Environment(SHINE): a protocol for a prospective study of diagnostic accuracy among adult hospital patients
}

To cite: Armitage LC, Mahdi A, Lawson BK, et al. Screening for Hypertension in the INpatient Environment(SHINE): a protocol for a prospective study of diagnostic accuracy among adult hospital patients. BMJ Open 2019;9:e033792. doi:10.1136/ bmjopen-2019-033792

- Prepublication history for this paper is available online. To view these files, please visit the journal online (http://dx.doi. org/10.1136/bmjopen-2019033792).

Received 22 August 2019 Revised 05 November 2019 Accepted 14 November 2019

Check for updates

(c) Author(s) (or their employer(s)) 2019. Re-use permitted under CC BY-NC. No commercial re-use. See rights and permissions. Published by BMJ.

${ }^{1}$ Nuffield Department of Primary Care Health Sciences, University of Oxford, Oxford, UK

${ }^{2}$ Institute of Biomedical Engineering, Department of Engineering Science, University of Oxford, Oxford, UK

${ }^{3}$ Nuffield Department of Clinical Neurosciences, University of Oxford, Oxford, UK

Correspondence to

Dr Laura C Armitage;

laura.armitage@phc.ox.ac.uk

\section{ABSTRACT}

Introduction A significant percentage of patients admitted to hospital have undiagnosed hypertension. However, present hypertension guidelines in the UK, Europe and USA do not define a blood pressure threshold at which hospital inpatients should be considered at risk of hypertension, outside of the emergency setting. The objective of this study is to identify the optimal in-hospital mean blood pressure threshold, above which patients should receive postdischarge blood pressure assessment in the community.

Methods and analysis Screening for Hypertension in the INpatient Environment is a prospective diagnostic accuracy study. Patients admitted to hospital whose mean average daytime blood pressure after 24 hours or longer meets the study eligibility threshold for mean daytime blood pressure $(\geq 120 / 70 \mathrm{~mm} \mathrm{Hg}$ ) and who have no prior diagnosis of, or medication for hypertension will be eligible. At 8 weeks postdischarge, recruited participants will wear an ambulatory blood pressure monitor for 24 hours. Mean daytime ambulatory blood pressure will be calculated to assess for the presence or absence of hypertension. Diagnostic performance of in-hospital blood pressure will be assessed by constructing receiver operator characteristic curves from participants' in-hospital mean systolic and mean diastolic blood pressure (index test) versus diagnosis of hypertension determined by mean daytime ambulatory blood pressure (reference test). Ethics and dissemination Ethical approval has been provided by the National Health Service Health Research Authority South Central-0xford B Research Ethics Committee (19/SC/0026). Findings will be disseminated through national and international conferences, peerreviewed journals and social media.

\section{INTRODUCTION}

Hypertension is the leading risk factor for death globally ${ }^{1}$ with $12.8 \%$ of annual mortality attributable to hypertension. ${ }^{2}$ A significant percentage of patients admitted to hospital have undiagnosed hypertension. ${ }^{34}$ However,

\section{Strengths and limitations of this study}

This is the first study to our knowledge to evaluate the diagnostic accuracy of a range of in-hospital blood pressure thresholds to identify the optimal blood pressure cut-off values for in-hospital hypertension screening.

- This is also the first study to our knowledge, to use real-time algorithms to screen hospital inpatients' electronic blood pressure data.

- Owing to the novel design of this study, the expected prevalence of hypertension at follow-up is unknown and has, therefore, been estimated to calculate the sample size.

unless a person has severely elevated blood pressure (BP), the clinical diagnosis of hypertension cannot be made in the in-hospital setting based on the UK, European or American guidelines. ${ }^{5-7}$ Indeed, increased BP values in hospital are frequently dismissed and additional potential reasons for this include clinicians attributing increased blood pressure values to anxiety, ${ }^{8}$ pain ${ }^{9}$ or white coat hypertension. ${ }^{10}$ However, the evidence suggests that patients with elevated BP recordings in hospital frequently remain hypertensive in the community. ${ }^{11-17}$ Despite this, referral for community follow-up of these patients to determine the presence or absence of persistent hypertension is poor. ${ }^{18-21}$

Untreated hypertension is associated with a progressive increase in $\mathrm{BP}$ that can become treatment resistant. ${ }^{22}$ Therefore, hospital detection and subsequent management of hypertension offer an important intervention opportunity to address this major cause of morbidity and mortality. 
In 2015, Oxford University Hospitals NHS Foundation Trust (OUHFT) introduced the NIHR-funded System for Electronic Notification and Documentation (SEND). ${ }^{23}$ SEND is a tailored software application, which links hospital bedside monitoring devices, including BP monitors, with a tablet computer for the manual recording of vital-sign observations of patients. ${ }^{24}$ Since this implementation, clinicians and researchers have been able to access and analyse patient observation data at a population as well as individual level. SEND has been used to record the vital-sign observations of more than 200000 patients so far and to link each patient's observations with their electronic patient record (EPR). ${ }^{24}$ This has paved the way for real time and automated recognition of patients whose observations indicate they may be at increased risk of undiagnosed hypertension.

The objective of this study is to identify the optimal in-hospital mean BP threshold, above which patients should receive postdischarge $\mathrm{BP}$ assessment in the community.

\section{METHODS AND ANALYSIS}

We prepared this protocol following the Standards for Reporting of Diagnostic Accuracy Studies (STARD) ${ }^{25}$ guidelines.

\section{Patient and public involvement}

The design of this study has been reviewed and supported by the patient and public representatives who have provided input on the research questions, data analysis, informational material for public engagement and the burden of the follow-up diagnostic testing from a patient's perspective. Their input led to additional steps in data analysis, refining of the informational material and modification of the follow-up plan to reduce the patient burden.

\section{Study design}

This is a UK single-centre, prospective cohort diagnostic accuracy study. Recruitment commenced in November 2019 and active follow-up will continue until January 2022.

\section{Study setting}

Recruitment for this study will take place in OUHFT, UK. Participants will be followed up in a community research clinic at 8 weeks $( \pm 4)$ following discharge from hospital to receive reference BP testing. Participant timeline is shown in figure 1.

\section{Study population}

We will recruit participants aged between 18 and 80 years, who have at least two BP measurements taken during daytime hours defined as 07:00:00 to 21:59:59 hours $^{26}$ and at least one BP measurement taken during night-time hours (22:00:00 to 06:59:59 hours). To be eligible, a patient's cumulative mean daytime systolic BP must be between $120 \mathrm{~mm} \mathrm{Hg}$ and $179 \mathrm{~mm} \mathrm{Hg}$ and cumulative mean daytime diastolic BP $\leq 109 \mathrm{~mm} \mathrm{Hg}$ for any given 24 hours interval since their admission to hospital following the first 24 hours of their admission (index test) - see the TEST METHODS section for more details. The mean daytime BP calculation must be based on a minimum of two blood pressure measurements made during day time hours. No lower eligibility threshold was set for the diastolic blood pressure in order that patients with isolated systolic hypertension would not be excluded. Patients with a diagnostic code for hypertension in their medical record, as classified by the 10th revision of the International Statistical Classification of Diseases and Related Health Problems (ICD-10) or BP lowering medication prescribed in their medication record will be excluded. Additional exclusion criteria include current, intended or recent pregnancy, cause for index admission being associated with end-organ damage related to severe hypertension (including but not limited to heart failure, myocardial infarction, stroke, hypertensive encephalopathy) and estimated glomerular infiltration rate of $<30 \mathrm{~mL} / \mathrm{min}$. Full inclusion and exclusion criteria are provided in table 1.

\section{Screening procedure}

The identification of patients whose BP exceeds the index test threshold will be automated using live, computerised algorithms. Blood pressure measurements in the OUHFT are made using automated oscillometric devices and the BP monitor readings (systolic and diastolic) are entered manually using the SEND computer tablet before automated transfer to the EPR by the SEND software app. ${ }^{23}$ The identification of patients whose BP measurements meet eligibility criteria will be computerised and automated. These automated systems for BP documentation and evaluating study inclusion criteria are intended to ensure that the measurement of the index test is objective and thus the clinical staff who perform the BP measurements can be considered blinded in the assessment of the index test. As this study is prospective in design, they will not be aware of the result of the reference standard.

\section{Participant sampling}

Sampling will be stratified to ensure that there is representation of patients with a full range of in-hospital BP profiles (according to predefined mean daytime systolic blood pressure, table 2). Patients who are considered eligible according to the screening algorithm will be approached by a clinical researcher who will undertake a further screen of patient eligibility. Patients who are in significant or uncontrolled pain will not be recruited unless and until, their pain improves. Pain scales will be collected at baseline for all recruited participants. Where there is insufficient study resource to enrol all recruitable patients, priority will be given to the strata with the least recruited patients. Recruitment will continue until the target recruitment number within 


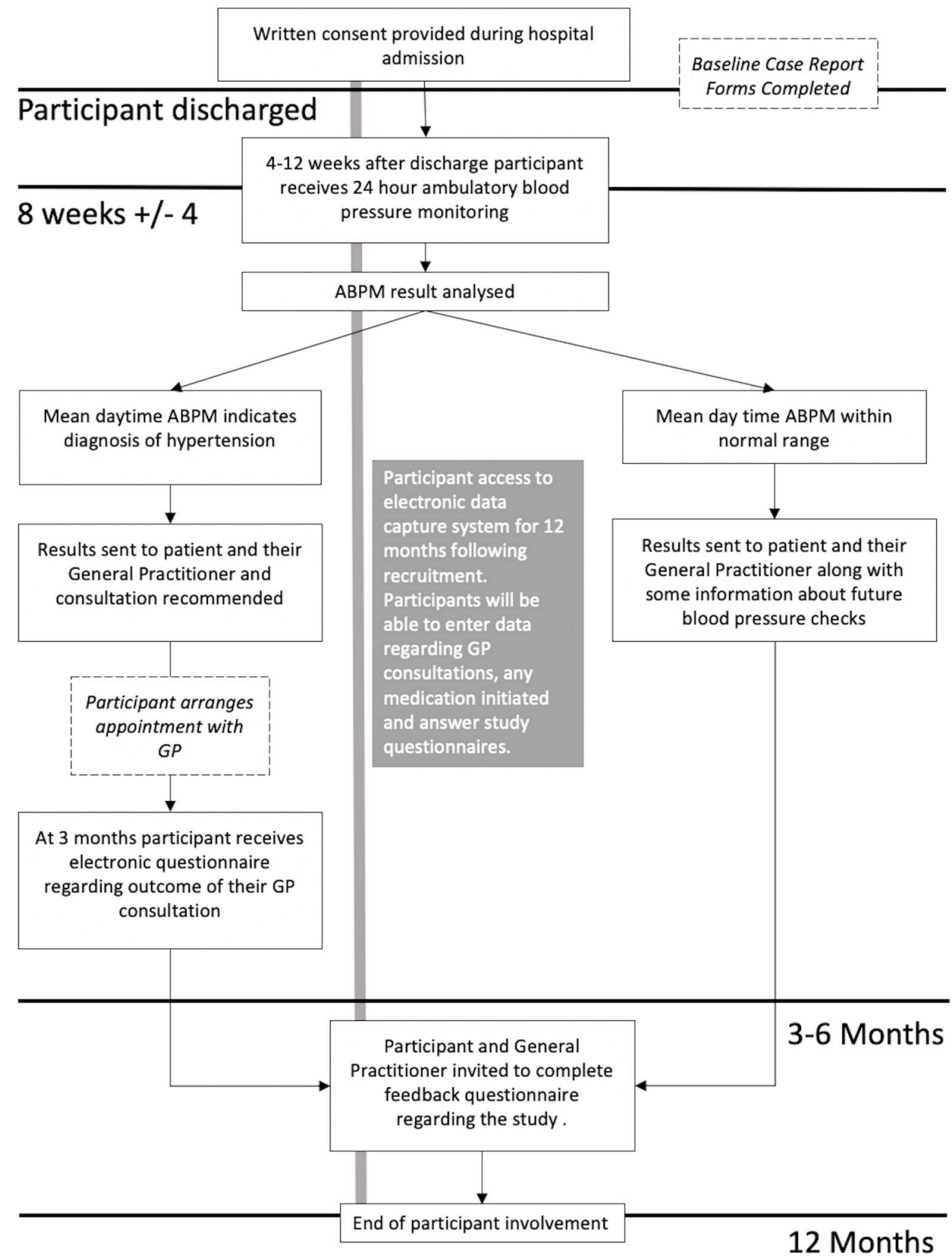

Figure 1 Participant timeline. ABPM, ambulatory blood pressure monitoring; GP, general practitioner.

each of the BP intervals is achieved. The prevalence of each systolic BP interval among the hospital population will be recorded within a screening log during the study period.

\section{Data collection}

Baseline data collection will be performed at the point of recruitment during the hospital admission, after the participant has provided informed, written consent. Custom-made baseline case report forms will be completed in the study data capture system for each participant by an appropriately qualified clinical researcher. The study data capture system will be electronic and accessible online. using a combination of data extracted from the EPR (eg, diagnostic codes, current medications, serum haematology and biochemistry results and ECG) and participant self-report (eg, ethnicity, family history, previous prescribed medications for hypertension). Any extreme values entered into the electronic data capture system will be inspected. A data monitoring committee has not been convened since there is no concealed allocation to treatment in this cohort study. Frequency and procedures for auditing study conduct are determined by the study sponsor (University of Oxford) based on a proportionate approach to risks. 
Table 1 Full inclusion and exclusion criteria

\section{Inclusion criteria}

Aged 18-80

Admitted to hospital for an acute or elective medical or surgical condition.

Have at least three in-hospital blood pressure recordings with two performed during day time hours and 1 performed during nighttime hours, over a minimum of 24 hours for the index admission.

Identified by the blood pressure algorithm for any 24 hours interval to have a mean blood pressure which meets the eligibility thresholds.

Registered with a general practitioner.

\section{Exclusion criteria}

Pre-existing diagnosis of hypertension or attending hospital with acute end-organ damage related to severe, undiagnosed hypertension.

Presence of atrial fibrillation or other pulse rate irregularity, which means ABPM is not appropriate.

Currently pregnant, within 3 months postpartum or planning pregnancy during study period.

Receiving treatments which might be used for the management of hypertension, for example, beta blockers for migraine, ACE inhibitors for renal disease.

Diagnosed with terminal illness or cognitive impairment.

Diagnosed with AKI on index admission or eGFR $<30 \mathrm{~mL} / \mathrm{min}$.

Cause for hypertension being toxicology, medical or recreational for example, amphetamines and their derivatives or alcohol withdrawal syndrome.

Postdischarge destination being another hospital or prison.

Receiving concomitant chemotherapy.

Already recruited to a separate hypertension study.

ABPM, ambulatory blood pressure monitoring; AKI, acute kidney injury; eGFR, estimated glomerular infiltration rate.

\section{TEST METHODS}

\section{Index diagnostic test}

The index test will be the first occurrence during the hospital stay of a cumulative mean daytime in-hospital $\mathrm{BP}$ reading $\geq 135 \mathrm{~mm} \mathrm{Hg}$ systolic or $\geq 85 \mathrm{~mm} \mathrm{Hg}$ diastolic. The systolic BP eligibility threshold has been defined as $10 \mathrm{~mm} \mathrm{Hg}$ below the index test threshold to understand the frequency of true and false negatives in order to calculate sensitivity and specificity of the diagnostic test.

$\mathrm{BP}$ measurements from the point of admission to the time of assessment by the algorithm (every 24 hours at midnight until day 10 of the admission or discharge, whichever occurred first) will be averaged to reduce the chances of a person becoming eligible by chance when compared with the alternative, of assessing a person's eligibility based on their mean day time BP for any 24 hours interval. Thus, for each patient at each time of assessment, we will compute the cumulative mean BP value using all the available daytime (7:00:00-21:59:59

\begin{tabular}{|c|c|}
\hline $\begin{array}{l}\text { Systolic blood pressure } \\
\text { interval }\end{array}$ & Target recruitment no \\
\hline $\begin{array}{l}120-129 \\
130-139 \\
140-149 \\
150-159 \\
160-179\end{array}$ & $\begin{array}{l}\text { Approximately equal } \\
\text { representation of participants } \\
\text { recruited to each of these } \\
\text { systolic blood pressure } \\
\text { intervals }\end{array}$ \\
\hline Total & 200 \\
\hline
\end{tabular}

hours) BP readings. The cumulative mean is defined here to be the mean of the mean daytime $\mathrm{BP}$ values, the latter being calculated for each of the days between the point of admission and the time of assessment. As the first occurrence of the test being passed is a necessary and sufficient condition, a patient will be recruited into the study even if their cumulative mean at discharge is below the index test thresholds $(\geq 135 \mathrm{~mm} \mathrm{Hg}$ systolic or $\geq 85 \mathrm{~mm} \mathrm{Hg}$ diastolic).

A day time definition was chosen as this study is being performed in a UK healthcare setting, where at present, night-time measurements are not used for the diagnosis of hypertension. ${ }^{27}$ The index test has been defined according to the UK National Institute for Health and Care Excellence (NICE) guideline definition of daytime hypertension. ${ }^{27}$

Additionally, a subgroup of participants will receive a research standard BP assessment in hospital, performed according to the standard recommended by the UK NICE guidelines. ${ }^{27}$ The BP will be measured using a validated and calibrated oscillometric device by a trained clinical researcher. BP will be measured with the patient seated and the arm outstretched and supported. BP will be measured in both arms and if a between-arm difference of $\geq 15 \mathrm{mg} \mathrm{Hg}$ is found, both measurements will be repeated. Where a difference persists, the arm with the highest measurement will be used for subsequent measurements. Two measurements will be taken in the selected arm; if the second measurement is substantially different from the first then a third measurement will be taken and the lowest of the second and third measurements will be recorded. 


\section{Reference standard diagnostic test}

The gold-standard diagnostic test for the presence of hypertension will be a daytime 24 hours ambulatory BP threshold of $\geq 135 \mathrm{~mm} \mathrm{Hg}$ systolic or $\geq 85 \mathrm{~mm} \mathrm{Hg}$ diastolic, which is the current UK NICE diagnostic threshold for ambulatory blood pressure monitoring (ABPM). ${ }^{5}$

ABPM will be performed with each participant at 8 weeks $( \pm 4)$ following discharge from hospital using a validated automated Mobil-O-Graph ABPM (IEM, Stolberg, Germany), calibrated to manufacturer standards. Should the participant have acquired a diagnosis of hypertension and initiated treatment for hypertension following hospital discharge but before they receive ABPM, they will not be eligible to proceed with ABPM. In this instance, a participant will be regarded as being reference test positive, having acquired a clinical diagnosis of hypertension. The monitor will be programmed to measure BP twice per hour during waking hours for (07:00-22:00 hours), and once per hour during the sleeping hours (22:0007:00 hours) for a period of 24 hours, according to the British and Irish Hypertension Society (BIHS) Standard Operating Procedure for performing ABPM. ${ }^{26}$ Adjustments will be made, for example, where a participant will be working a shift pattern during the period of wear. All participants will be asked to log their sleeping and waking times in an ABPM diary and these will be used to analyse the ABPM results. According to the BIHS standard operating procedure for ABPM, a minimum of 14 waking time ABPM measurements must be successful to calculate the mean daytime BP. Where fewer than 14 of the waking time ABPM measurements are successful, the participant will be asked to wear the monitor for a further period in order to collect adequate data. In the instance of two episodes of ABPM not collecting sufficient data, the participant and their registered general practitioner (GP) will be informed along with recommendation that the participant arrange an appointment with their GP for further evaluation of their BP. The participant will continue to be followed up remotely through the online data capture system for up to 52 weeks from recruitment (figure 1). Links to the questionnaires will be sent to participants by email and we will collect data regarding BP-related health outcomes, the prescription of antihypertensive medications and repeat blood pressure measurements. At 10 years, data will be obtained from the Office of National Statistics and Hospital Episode Statistics Databases regarding mortality and cardiovascular health-related outcomes.

The fitting and removal of the ABPM will be performed by a qualified clinical research nurse who is appropriately trained in the National Institute for Health's Good Clinical Practice and to perform ABPM. At the time of removal of the monitor, the electronic ABPM report will be downloaded to the study data capture system and interpreted by the research nurse. As the reference test procedure is objective through the use of an oscillometric, automated ABPM, the research nurse will not be blinded to the result of the index BP test.
The same subgroup of 50 participants who received a standardised research BP assessment in hospital will receive a standardised research clinic blood pressure on ABPM fitting. The procedure for measuring and recording the BP will be exactly the same as described for the hospital setting, according to that recommended by the UK NICE guidelines. ${ }^{27}$

\section{STATISTICAL METHODS}

\section{Sample size}

Owing to the novel design of this study, estimates of expected prevalence of hypertension at follow-up have been used to calculate sample size. From a retrospective analysis of in-hospital OUHFT BP data, we estimate that 3000 patients per annum will be eligible for study inclusion (Mahdi, A, 2019; Prevalence of hypertension and undiagnosed hypertension in a large inpatient population). Previous studies have used an in-hospital BP eligibility threshold $(140 / 90 \mathrm{~mm} \mathrm{Hg})$ and revealed that approximately half of the patients with a BP above the threshold have hypertension at follow-up. ${ }^{28} 29$ Although this study uses a lower eligibility BP threshold of $120 / 70 \mathrm{~mm} \mathrm{Hg}$; we estimate half of the patients will have hypertension at follow up owing to the stratification used for recruitment. Therefore, a sample size of 196 participants has been estimated to permit detection of a $50 \%$ rate of hypertension at follow-up, with a $95 \%$ confidence interval width of approximately $\pm 7 \%$. Screening logs will be recorded for both the algorithm and the manual screening process to understand the prevalence of patients falling in each of the in-hospital BP intervals against which recruitment will be stratified.

\section{Baseline characteristics}

Clinical and demographic characteristics of the study population will be reported, including age, sex, presenting complaint, comorbidity, current treatments (including oral steroidal and non-steroidal anti-inflammatory medications), pain score (using an 11-point Likert scale where $0=$ no pain and $10=$ maximum possible pain) for the preceding 24 hours to recruitment and the Six-Item State Anxiety Scale. ${ }^{30}$ The number of participants who complete each stage of the protocol will be reported, including those who complete successful ABPM, with reasons for failed testing where appropriate. Recruitment and study completion figures will be reported using a participant flow diagram. Interpretation of the ABPM result will be performed by the researchers who are blind to the participant's index BP test.

\section{Index test performance}

Diagnostic performance of in-hospital BP will be assessed by constructing receiver operator characteristic (ROC) curves from participants' in-hospital mean systolic or mean diastolic BP (index test) versus diagnosis of hypertension determined by mean daytime ambulatory systolic and diastolic BP (reference test). At first instance, a complete-case analysis will be performed by excluding 
Table 3 European Society of Cardiology/European Society of Hypertension ${ }^{6}$ and American College of Cardiology ${ }^{7}$ diagnostic thresholds for hypertension

\begin{tabular}{|c|c|c|c|c|}
\hline \multirow{2}{*}{$\begin{array}{l}\text { ABPM diagnostic } \\
\text { thresholds for } \\
\text { hypertension }\end{array}$} & \multicolumn{2}{|c|}{$\begin{array}{l}\text { European Society of Cardiology/European Society of } \\
\text { Hypertension }\end{array}$} & \multicolumn{2}{|c|}{ American College of Cardiology } \\
\hline & Systolic & Diastolic & Systolic & Diastolic \\
\hline Night & 120 & 70 & 110 & 65 \\
\hline Day & 135 & 85 & 130 & 80 \\
\hline
\end{tabular}

ABPM, Ambulatory Blood Pressure Monitoring.

participants without an observed test result. At the second instance, for participants with missing ABPM values, the data will be imputed. The two approaches will be compared and the number of cases with missing values will be reported, together with detailed description of the strategy for handling the missing data. The area under the ROC curve will be calculated to estimate the discriminatory power of the diagnostic test. ${ }^{31}$ Potential diagnostic BP thresholds will then be identified from the ROC curve. $^{31} 32$ The diagnostic ability of each potential cutoff threshold will be evaluated using two-by-two tables ${ }^{32}$ to compare sensitivity and specificity of each potential threshold. Confidence intervals will be reported for each value. Reproducibility of the index test and variability in diagnostic accuracy will be analysed using subsequent available periods of the hospital admission in which participants' mean in-hospital BP values were at or above the recruitment threshold.

\section{Subgroup analyses}

Logistic regression analyses will be performed to estimate whether the diagnostic performance of in-hospital mean BP is influenced by participant baseline characteristics, including age, sex body mass index and comorbidity, pain, anxiety and in-hospital prescriptions such as oral steroid medications and non-steroidal anti-inflammatory medications. Further subgroup analyses will include whether the index admission was scheduled or unscheduled and whether the admitting specialty was medical or surgical.

\section{Additional analyses}

Once the optimal thresholds for the in-hospital screening for hypertension have been identified, diagnostic accuracy of the thresholds at successive 24 hours intervals of patient admission will be estimated. While the reference test for this study is based on the current UK diagnostic criteria for the diagnosis of hypertension, which concerns daytime BP measurements only, there is increasing evidence in the literature to support the association between nocturnal hypertension and cardiovascular risk. ${ }^{334}$ Therefore, additional analyses will be performed incorporating night-time measurements and using the European and American diagnostic thresholds for hypertension as shown in table $3 .{ }^{67}$

\section{ETHICS AND DISSEMINATION}

Any planned protocol modifications will receive further sponsor and ethical review and require approval prior to implementation. Any approved modifications will be communicated in the ISRCTN Registry where the study is registered (Identification number: ISRCTN80586284). All participants will be required to give informed, written consent and will be advised that they can withdraw from the study at any time and without giving reason. Consent will be obtained by a trained clinical researcher.

We do not anticipate any harms from the use of ABPM, which is already a standard procedure for the diagnosis of hypertension in UK primary care, and therefore, this study does not carry any significant risk to participants. The measurement of ambulatory BP among the participants of this study is in addition to usual care and may be considered beneficial if we detect the presence of undiagnosed hypertension earlier than may have otherwise been the case. If at any point a participant is found to have severely raised blood pressure, their responsible hospital care team or GP will be informed, following study standard operating procedures, according to whether severely elevated BP was observed in the hospital or community, respectively. Any adverse events, which arise as a result of performing ABPM, will be reported. The participant and their GP will both be informed of the ABPM result and any decision to initiate treatment will be made between the patient and their GP in line with standard care.

Data will be analysed by researchers based in the University of Oxford (Nuffield Department of Clinical Neurosciences, Nuffield Department of Primary Care Health Sciences and Institute of Biomedical Engineering).

\section{Dissemination}

Findings from this study will be disseminated through peer-reviewed journals, conferences, public engagement activities and online. Participants will be able to access results online.

\section{Discussion}

The Screening for Hypertension in the INpatient Environment study will be the first study to our knowledge, to evaluate the diagnostic accuracy of in-hospital BP measurements on a continuous scale and therefore identify the optimal BP cut-off values for in-hospital 
hypertension screening. A recent systematic review indicates this will be the first study to use real-time algorithms to screen hospital inpatients' electronic BP data. ${ }^{17}$ The automated detection and subsequent treatment of previously undiagnosed hypertension has the potential to prevent the development of serious hypertension-related diseases.

The study is limited by uncertainty in the expected prevalence of hypertension at follow-up, which has been used to estimate the required sample size.

\section{Twitter Laura C Armitage @LauraArmitage5}

Contributors PJW is the principal investigator. LCA, PJW, AJF, AM, CR and LT made substantial contributions to the conception and design of the project. BKL has contributed substantially to protocol development. TF provided advice on statistical methodology. All authors contributed to and revised the final manuscript.

Funding This study is funded by the National Institute for Health (NIHR) Oxford Biomedical Research Centre (BRC) Technology and Digital Health theme. The sponsor is the University of Oxford. AJF is an NIHR Senior Investigator. The sponsor has provided ethical and legal guidance in study conduction.

Disclaimer The views expressed are those of the authors and not necessarily those of the NIHR or the Department of Health and Social Care. Neither the funder nor sponsor have had or will have, any role in study design, collection, management, analysis, interpretation of data or writing of the report.

Competing interests PJW and LT are the Chief Medical Officer and R\&D Director for Sensyne Health and hold share options in the company. Their University departments receive research funding from Sensyne Health.

Patient consent for publication Not required.

Ethics approval This study (Protocol V.1.7) has been given ethical approval from the NHS Health Research Authority South Central-0xford B Research Ethics Committee (19/SC/0026).

Provenance and peer review Not commissioned; externally peer reviewed.

Open access This is an open access article distributed in accordance with the Creative Commons Attribution Non Commercial (CC BY-NC 4.0) license, which permits others to distribute, remix, adapt, build upon this work non-commercially, and license their derivative works on different terms, provided the original work is properly cited, appropriate credit is given, any changes made indicated, and the use is non-commercial. See: http://creativecommons.org/licenses/by-nc/4.0/.

\section{ORCID iDs}

Laura C Armitage http://orcid.org/0000-0002-5009-4899

Andrew J Farmer http://orcid.org/0000-0002-6170-4402

Peter J Watkinson http://orcid.org/0000-0003-1023-3927

\section{REFERENCES}

1 Campbell NRC, Lackland DT, Niebylski ML, et al. High blood pressure: why prevention and control are urgent and Important-A 2014 fact sheet from the world hypertension League and the International Society of hypertension. J Clin Hypertens 2014:16:551-3.

2 World Health Organisation. Global Health Observatory (GHO) data: Raised blood pressure. WHO Published Online First, 2015. Available: http://www.who.int/gho/ncd/risk_factors/blood_pressure_ prevalence_text/en/ [Accessed 27 Feb 2018].

3 Shankar BS, Russell RP, Southard JW, et al. Patterns of care for hypertension among hospitalized patients. Public Health Rep 1982;97:521-7.

4 Greenland P, Levenkron JC, Smith RM. Hospitalization as an opportunity to improve hypertension recognition and control. Med Care 1987;25:717-23.

5 National Institute for Health and Care Excellence. Clinical guideline CG127. Hypertension in adults: diagnosis and management, 2011. Available: https://www.nice.org.uk/guidance/cg127 [Accessed 2 Aug 2018].

6 Williams B, Mancia G, Spiering W, et al. 2018 ESC/ESH guidelines for the management of arterial hypertension. J Hypertens 2018;36:1953-2041.
7 Whelton PK, Carey RM, Aronow WS, et al. 2017 ACC/AHA/AAPA ABC/ACPM/AGS/APhA/ASH/ASPC/NMA/PCNA guideline for the prevention, detection, evaluation, and management of high blood pressure in adults. J Am Coll Cardiol 2018;71:e127-248.

8 Backer HD, Decker L, Ackerson L. Reproducibility of increased blood pressure during an emergency department or urgent care visit. Ann Emerg Med 2003;41:507-12.

9 Tanabe P, Cline DM, Cienki JJ, et al. Barriers to screening and intervention for ED patients at risk for undiagnosed or uncontrolled hypertension. J Emerg Nurs 2011;37:17-23.

10 Khan T V, Khan SS-S, Akhondi A, et al. White coat hypertension: relevance to clinical and emergency medical services personnel. Medscape Gen Med 2007;9:52.

11 EUROASPIRE Study Group. EUROASPIRE: a European Society of cardiology survey of secondary prevention of coronary heart disease: principal results. Eur Heart J 1997;18:1569-82.

12 EUROASPIRE II Study Group. Lifestyle and risk factor management and use of drug therapies in coronary patients from 15 countries; principal results from EUROASPIRE II Euro heart survey programme. Eur Heart J 2001;22:554-72.

13 Jankowski P, Kawecka-Jaszcz K, Bilo G, et al. Determinants of poor hypertension management in patients with ischaemic heart disease. Blood Press 2005;14:284-92.

14 Conen D, Martina B, Perruchoud AP, et al. High prevalence of newly detected hypertension in hospitalized patients: the value of inhospital 24-h blood pressure measurement. $J$ Hypertens 2006;24:301-6.

15 Kotseva K, Wood D, Backer GD, et al. EUROASPIRE III: a survey on the lifestyle, risk factors and use of cardioprotective drug therapies in coronary patients from 22 European countries. Eur J Cardiovasc Prev Rehabil 2009;16:121-37.

16 Kotseva K, Wood D, De Bacquer D, et al. EUROASPIRE IV: a European Society of cardiology survey on the lifestyle, risk factor and therapeutic management of coronary patients from 24 European countries. Eur J Prev Cardiol 2016;23:636-48.10.1177/2047487315569401

17 Armitage LC, Whelan ME, Watkinson PJ, et al. Screening for hypertension using emergency department blood pressure measurements can identify patients with undiagnosed hypertension: a systematic review with meta-analysis. J Clin Hypertens 2019:jch.13643.

18 Tilman K, DeLashaw M, Lowe S, et al. Recognizing asymptomatic elevated blood pressure in ED patients: how good (bad) are we? Am $J$ Emerg Med 2007;25:313-7.

19 Baumann BM, Cienki JJ, Cline DM, et al. Evaluation, management, and referral of elderly emergency department patients with elevated blood pressure. Blood Press Monit 2009;14:251-6.

20 Lehrmann JF, Tanabe P, Baumann BM, et al. Knowledge translation of the American College of emergency physicians clinical policy on hypertension. Acad Emerg Med 2007;14:1090-6.

21 Baumann BM, Cline DM, Cienki JJ, et al. Provider self-report and practice: reassessment and referral of emergency department patients with elevated blood pressure. Am J Hypertens 2009;22:604-10.

22 National Institute for Health and Clinical Excellence. Guideline scope: hypertension in adults (update), 2017. Available: https://www.nice. org.uk/guidance/GID-NG10054/documents/draft-scope [Accessed 27 Feb 2018]

23 Wong D, Bonnici T, Knight J, et al. Send: a system for electronic notification and documentation of vital sign observations. BMC Med Inform Decis Mak 2015;15:68.

24 Wong D. SEND: system for electronic notification and documentation. Available: http://www.send-system.co.uk/about.html [Accessed 2 Aug 2018].

25 Cohen JF, Korevaar DA, Altman DG, et al. Stard 2015 guidelines for reporting diagnostic accuracy studies: explanation and elaboration. BMJ Open 2016;6:e012799.

26 British and Irish Hypertension Society. Standard operating procedure for ambulatory blood pressure monitoring (ABPM), 2011. Available: https://bihsoc.org/resources/bp-measurement/abpm/ [Accessed 11 Aug 2018].

27 National Institute for Health and Care Excellence. Hypertension in adults: diagnosis and management. NICE, 2019. Available: https:// www.nice.org.uk/guidance/ng136 [Accessed 16 Oct 2019].

28 Tsoi LCH, Tung CCH, Wong ELY. Nurse-Led hypertension referral system in an emergency department for asymptomatic elevated blood pressure. Hong Kong Med J 2012;18:201-6.

29 Arhami Dolatabadi A, Motamedi M, Hatamabadi H, et al. Prevalence of undiagnosed hypertension in the emergency department. Trauma Mon 2014;19:e7328. 
30 Marteau TM, Bekker $\mathrm{H}$. The development of a six-item short-form of the state scale of the Spielberger State-Trait anxiety inventory (STAI). Br J Clin Psychol 1992;31:301-6.

31 Greiner M, Pfeiffer D, Smith RD. Principles and practical application of the receiver-operating characteristic analysis for diagnostic tests. Prev Vet Med 2000;45:23-41.

32 Hajian-Tilaki K. Receiver operating characteristic (ROC) curve analysis for medical diagnostic test evaluation. Casp J Intern Med 2013;4:627-35.
33 Fagard RH, Thijs L, Staessen JA, et al. Night-day blood pressure ratio and dipping pattern as predictors of death and cardiovascular events in hypertension. J Hum Hypertens 2009;23:645-53.

34 Tsioufis C, Andrikou I, Thomopoulos C, et al. Increased nighttime blood pressure or nondipping profile for prediction of cardiovascular outcomes. J Hum Hypertens 2011;25:281-93. 